\title{
Comparison of Outcomes and Complications Among Various Surgical Treatments for Vertebral Compression Fractures: a Retrospective Study.
}

Kuei-Lin Yeh

Shin Kong Wu Ho-Su Memorial Hospital

Szu-Hsien Wu

Taipei Veterans General Hospital

Shing-Sheng Wu ( $\nabla$ raysswu@gmail.com )

Shin Kong Wu Ho-Su Memorial Hospital

Sheng-Mou Hou

Shin Kong Wu Ho-Su Memorial Hospital

Research article

Keywords: vertebral compression fracture, vertebroplasty, kyphoplasty, osteoporosis, SpineJack

Posted Date: January 20th, 2021

DOI: https://doi.org/10.21203/rs.3.rs-150235/v1

License: (a) (i) This work is licensed under a Creative Commons Attribution 4.0 International License. Read Full License 


\section{Abstract}

\section{Background}

Osteoporosis with vertebral compression fractures is increasingly common in the elderly. As no studies have compared the safety and efficacy of surgical techniques for the treatment of such fractures, we retrospectively compared vertebroplasty, balloon kyphoplasty, and kyphoplasty with SpineJack or an intravertebral expandable pillar (IVEP). In this study, we retrospectively compared the safety and efficacy, including visual analog scale scores for pain, kyphotic angle, average body height, rate of cement leakage, and occurrence of adjacent vertebral compression fractures of each surgical intervention mentioned above at Shin-Kong Wu Ho-Su Memorial Hospital. All patients underwent surgical treatment under Dr. Wu.

\section{Methods}

We retrospectively analyzed 10 years of data of 354 patients with vertebral compression fractures, randomly dividing them into five groups. All these patients were diagnosed with fresh compression fracture, defined as signal changes on T1 phase by magnetic resonance imaging examination. Their visual analog scale scores for pain, kyphotic angle, average body height, rate of cement leakage, and occurrence of adjacent vertebral compression fractures were followed up for 1 year. ANOVA, the post hoc Bonferroni test, and Fisher's exact probability test were used for statistical analyses.

Results

All pain scores significantly improved 12 months postoperatively; however, there was no significant difference between the groups. Kyphoplasty with SpineJack significantly reduced the kyphotic angle, restoring the vertebral body height; adjacent compression fracture rate was highest in the vertebroplasty group $(p=0.020)$. The method with the lowest cement leakage rate remains unidentified due to the small sample size; however, kyphoplasty with SpineJack, intravertebral expandable pillar, and vesselplasty resulted in lower rates than balloon kyphoplasty or vertebroplasty.

\section{Conclusions}

Vertebroplasty and kyphoplasty were excellent treatments for vertebral compression fractures, with no differences in pain relief. Kyphoplasty with SpineJack entailed a lower risk of cement extravasation, resulting in greater vertebral body height restoration and kyphotic reduction than other groups. While the safest method remains unclear, Kyphoplasty with SpineJack, intravertebral expandable pillar, and vesselplasty resulted in low rates of cement leakage. Kyphoplasty with intravertebral expandable pillar resulted in the lowest adjacent compression fracture rate.

\section{Background}


Osteoporosis in the elderly is becoming increasingly common with the increasing age of the population and affects approximately $35 \%$ of women over the age of 65 years [1]. Vertebral compression fractures (VCFs) represent a common problem in osteoporosis [2]. VCFs destabilize the vertebral body, whereas back pain is attributed to macro- and micromotion at the fracture site. In addition to back pain, the sequelae of VCFs are sometimes related to kyphotic angulation of the spine, which can diminish forced vital capacity [3, 4].

Management of VCFs includes both conservative treatments and surgical interventions. Conservative treatments include bed rest or activity modification, analgesics, and bracing; however, for most patients, conservative treatment is ineffective and unsatisfactory [5]. The kyphotic change in spine alignment reduces the lung cavity and shifts the center of gravity forward; thus, for elderly patients, bed rest for long periods is associated with complications such as pneumonia, urinary infections, and deep vein thrombosis $[6,7]$ Ultimately, conservative treatment does not correct spinal malalignment.

Surgical treatments for VCFs are increasingly important as they result in improvements in back pain and function, as well as quality of life following treatment [8]. There are several reported real-world practice and management options for VCFs, such as balloon kyphoplasty (KP), KP with SpineJack, KP with an intravertebral expandable pillar (IVEP), and vesselplasty. However, to date, no studies have compared their efficacy and safety; this was the main purpose of our study. The study was a retrospective study. All patients underwent surgical treatment by the same doctor, professor Shing-Sheng Wu. Emphasis was placed on the visual analogue scale (VAS) scores for pain, kyphotic angle (KA), average body height $(\mathrm{AVBH})$, complications, and occurrence of adjacent vertebral compression fractures during the 1-year follow-up.

\section{Methods}

\section{Patient population}

We retrospectively collected the data of 354 patients with VCFs treated between 2009 and 2019. All patients were admitted to the hospital due to severe back pain or intense aggravation of chronic back pain and were diagnosed using magnetic resonance imaging (MRI), performed within 4 weeks prior to hospitalization. Only patients with acute ( $<2$ weeks), subacute ( 2 weeks to 3 months), or nonunion VCFs, diagnosed via MRI signal alteration in the vertebral body, underwent vertebroplasty (VP) or KP; the same doctor performed surgical treatment. The levels of VCFs ranged from T6 to L4. The 354 patients were randomly divided into five groups: VP (88 patients; 20 males, 68 females), balloon KP (124 patients; 45 males, 79 females), KP with an IVEP (46 patients; 13 males, 33 females), KP with a vessel (36 patients; 11 males, 25 females), and KP with SpineJack (Vexim, Balma, France) (60 patients; 18 males, 42 females) (Table 1). All patients were aged $45-86$ years. The surgical treatments were all performed bipedicularly. The inclusion criterion was that patients continued postoperative follow-up examination at our outpatient clinic for at least 1 year. Patients without regular orthopedic outpatient follow-up for a minimum of 1 year were excluded. 
Table 1

Patient data and cement volume for each different surgical intervention

\begin{tabular}{|c|c|c|c|c|}
\hline & No. of patients & $\begin{array}{l}\text { Age } \\
\text { (years) }\end{array}$ & $\begin{array}{l}\text { Gender } \\
(M / F)\end{array}$ & Volume of cement \\
\hline VP & 88 & $\begin{array}{l}74.3 \pm 6.4 \\
(50-86)\end{array}$ & $20 / 68$ & $\begin{array}{l}3.2 \\
(1.0-7.0)\end{array}$ \\
\hline KP-Balloon & 124 & $\begin{array}{l}72.3 \pm 7.6 \\
(50-85)\end{array}$ & $45 / 79$ & $5.5 \pm 0.6$ \\
\hline KP-IVEP & 46 & $\begin{array}{l}72.7 \pm 6.4 \\
(50-90)\end{array}$ & $13 / 33$ & --- \\
\hline KP-Vessel & 36 & $\begin{array}{l}77.9 \pm 9.5 \\
(65-89)\end{array}$ & $11 / 25$ & $5.7 \pm 2.0$ \\
\hline KP-SJ & 60 & $\begin{array}{l}65.9 \pm 1.9 \\
(45-87)\end{array}$ & $18 / 42$ & $4.8 \pm 0.4$ \\
\hline
\end{tabular}

[Table 1 near here]

\section{VAS score}

VAS was used to indicate "pain at rest", with 0 being no pain and 10 being the worst pain ever experienced. The VAS score was evaluated based on the patient's subjective feelings. The ratio of postoperative to preoperative VAS score was calculated; the evaluation was performed 12 months postoperatively.

\section{Average body height}

We defined average body height (AVBH) as the average of the anterior edge of the vertebral body height and posterior edge of the vertebral body height in the lateral view of the spinal radiographs. The ratio of preoperative $\mathrm{AVBH}$ at 1 year postoperatively was calculated.

\section{Kyphotic angle}

The KA was used to quantify the magnitude of vertebral body wedge deformities. We drew lines along the vertebral upper endplate and lower endplate using lateral spine radiographs. We then measured the angle between the two lines and defined it as the KA, measured 1 year postoperatively and preoperatively; the ratio of these two data points was then recorded.

\section{Adjacent vertebral compression fracture}


We counted the occurrence of adjacent VCFs 1 year postoperatively; Fisher's exact probability test was applied. The null hypothesis was that there would be no difference between the groups; once the $p$-value was less than 0.05 , the null hypothesis was rejected.

\section{Cement leakage}

In this study, we recorded the symptoms of patients with cement leakage events and divided them into symptomatic or asymptomatic episodes.

\section{Statistical analysis}

ANOVA with the post hoc Bonferroni test was used to compare differences in baseline characteristics and percent changes in VAS and AVBH at 1 year among the four groups. One-way ANOVA was used to examine the significance of longitudinal changes in VAS scores at 3, 6, and 12 months. A significance level of $p<0.05$ was used for all comparisons.

Fisher's exact probability test was performed to determine the prevalence of adjacent vertebral compression fractures. The null hypotheses were rejected if $p$-values were less than 0.05 , analyzed using SPSS 12.0 statistical software.

\section{Results}

\section{VAS score}

Pain relief was measured using a VAS score ranging from 0 (no pain) to 10 (the worst pain ever). A 1-year follow-up was completed for all patients. Compared with baseline scores, we found improvements in VAS scores after VP or KP 12 months postoperatively. One-way ANOVA revealed no significant difference $\left(P_{12 \text { th month }}=0.325\right)$ among the various surgical treatments in terms of postoperative VAS scores (Figure 1).

\section{Average body height}

Statistical analysis for AVBH was performed using the ratio of postoperative to preoperative AVBH via one-way ANOVA, which revealed a significant difference $(p=0.020)$, and was followed by post hoc Bonferroni analysis. Significant differences were observed in the VP and KP with SpineJack groups when compared with other groups in the post hoc analysis (both groups were $\mathrm{p}<0.05$ ) (Figure 2).

\section{Kyphotic angle}

One-way ANOVA showed $p=0.028$, and the VP and KP with SpineJack groups showed a significant difference in comparison with the other groups in the post hoc Bonferroni analysis (both groups were $\mathrm{p}<0.05$ ) (Figure 3).

\section{Adjacent VCF}


Within 1 year, the number of adjacent VCFs was $15(15 \%), 4(6.7 \%), 7(5 \%), 2(5 \%)$, and 2 (4\%) patients in the VP, KP with SpineJack, balloon KP, patients in the KP with a vessel, and KP groups, respectively (Figure 4). Fisher's exact probability test was performed. The $p$-value was $<0.050(p=0.020)$; thus, the null hypothesis was rejected. Nevertheless, once we excluded the value of the VP group, a Fisher's exact probability test was performed. The p-value was 0.93 , which is substantially greater than 0.05 .

\section{Cement leakage}

Cement leakage is the most common complication among VCF surgical treatments. In the VP group, there were 24 cases of cement leakage, with symptomatic problems occurring in 8 patients. In the balloon KP group, cement leakage occurred in 30 cases, with 7 symptomatic cases. One patient (1.7\%) who underwent KP with SpineJack treatment suffered from non-symptomatic cement leakage. Failure of symptom relief occurred in only one patient (2\%) who underwent KP with IVEP due to implant migration. Similarly, one patient who underwent KP with vessel treatment suffered from symptomatic problems due to cement leakage.

\section{Ethical approval}

The requirement for written consent was waived owing to the retrospective study design. Ethical approval was obtained from the institutional review board of Shin-Kong Wu Ho-Su Memorial Hospital, Taiwan (approval number, 20200608R).

\section{Discussion}

VCFs cause debilitating back pain, and as a result of population aging, the incidence of these fractures increases [9]. Conservative treatment for compression fractures has not been suggested, as it fails to restore the vertebral body to its origin height. Additionally, pain and spinal deformity are not improved or corrected, and the domino effect follows rapidly after the first VCF [10]. This effect is a direct consequence of the mechanical variations that affect the spine when physiological curves are modified [11]. Treatment of VCFs with VP or KP maintains the integrity of adjacent structures and reduces the likelihood of a domino effect [10]; thus, the role of surgical treatment has increased.

Currently, percutaneous cement augmentation procedures are gaining popularity [12]. VP or KP can provide immediate effective pain relief for patients with VCFs [13]. To date, there have been several papers regarding minimally invasive cement augmentation procedures published. Nevertheless, no study has compared the efficacy and safety of these procedures; therefore, we compared VP, balloon KP, KP with an IVEP, KP with a vessel, and KP with SpineJack, performed via a bipedicular transpedicular approach by the same surgeon in five similar groups of patients.

The VAS is the most common pain measurement method. Several studies have found a significant improvement in pain after VP or KP $[14,15]$. These results are in line with those of our studies; we found that both VP and KP were effective at reducing pain, as measured by the VAS pain scale 12 months 
postoperatively. The VAS score was not significantly different between the KP and VP groups at 12 months postoperatively. The results showed that once polymethylmethacrylate (PMMA) was injected into the vertebral body to stabilize the fracture, the pain relief was significantly increased, regardless of the type of surgical intervention.

$\mathrm{KP}$ with SpineJack provides the best performance for AVBH restoration 1 year postoperatively. SpineJack is a permeant titanium implant designed to restore vertebral height through an endplate distraction device by expanding like a small jack; it is equipped with a mechanical opening, ensuring a gradual and controlled vertebral fracture reduction. This technique allows good reconstruction of the anterior column and restores vertebral height [16].

The KA evaluates the reduction in kyphosis. In our study, VP was unfavorable for kyphotic reduction due to the lack of effective strength acting on the upper and lower endplates. VP requires cement injection at higher pressures and hence incurs a higher risk of cement extravasation than KP and merely eliminates the deformity, without reduction of kyphosis $[14,17]$.

Berlemann et al. reported a mean kyphotic reduction of approximately $8^{\circ}$ in a group of 27 patients after KP [18], whereas Theodorou et al. revealed an average KA correction of $9^{\circ}$ [14]. Weisskopf et al. showed that the reduction in kyphotic deformity was only achieved in four cases (average, $8.5^{\circ}$ ) in a 22-patient trial [19]. In contrast, the corrections of KAs in our study were $14.2^{\circ}, 16.0^{\circ}, 15.8^{\circ}$, and $14.2^{\circ}$ in balloon, IVEP, vessel, and SpineJack KP, respectively, 1 year after surgical treatment; our kyphotic correction was substantially higher than those reported in previous studies. We were unable to discriminate between the acute VCFs from old VCFs using only conventional two-plane X-ray in an erect position instead of MRI. In the acute or subacute phase of VCF, edema was noted during replacement of the normal vertebral body bone marrow in a benign compression fracture, resulting in hypointensity on T1-weighted images and hyperintensity on T2-weighted images. The nonunion VCF presented a confined high intensity or a diffuse low-intensity area on T2-weighted MRI, found to be of significant importance $[20,21]$. The more recent the fracture, the better the kyphotic reduction after KP [22]. In contrast to previous studies, patients with a diagnosis of acute, subacute, or nonunion VCFs underwent surgical treatment in our study. Therefore, we gained better satisfaction from patients and better postoperative correction of the kyphotic deformity.

Of the five groups, adjacent VCF episodes occurred most commonly in the VP group. KP requires a larger injection pressure, creating a cancellous fill effect with PMMA, finally leading to better spinal biomechanical stability; as a result, the risk of secondary vertebral fractures decreases [5].

To date, it is not clear how new vertebral fractures occur in adjacent vertebrae. Some studies have shown that the pressure on adjacent vertebrae was increased as a result of injected cement [23]. Both VP and KP reduced swelling of the vertebral endplate and vertebral joint mobility, causing swelling of the adjacent vertebrae and increasing the risk of adjacent vertebral fracture [24]. In this study, we found that the highest rate $(17 \%)$ of adjacent VCFs occurred in the VP group. 
In the KP group, although the null hypothesis was not rejected, the difference may be related to the small amount of data. It appeared that the prevalence of adjacent VCFs within 1 year was lowest in the KP with IVEP group. Generally, KP with balloon, vessel, or SpineJack, as well as VP, are completed by filling the vertebral body with PMMA; only KP with an IVEP is associated with bone healing. The healed vertebrae following IVEP treatment have similar bone mineral densities to the adjacent vertebrae. The fractured vertebrae filled with PMMA was stiffer than the adjacent level vertebrae, explaining the lower adjacent VCF rate in the KP with IVEP group.

Complications of percutaneous VP or KP are fairly common, and in most cases, they are asymptomatic $[25,26]$. The most common complications are cement leakage due to the fact that osteoporosis increases the vulnerability of the vertebral bone structure; extraction of the endplate when performing KP may further displace the fractured fragment $[27,28]$, which can migrate into veins, paravertebral soft tissue, intervertebral discs, or the spinal canal, affecting the foraminal area or epidural space. In the VP and balloon KP groups, there was a higher percentage of cement leakage, 24 cases (16 asymptomatic, and 8 symptomatic) and 30 cases (23 asymptomatic, 7 symptomatic), respectively. Cement leakage or implant migration occurred less frequently in the KP with IVEP, vessel, and SpineJack groups.

With VP, the cement was injected into the vertebral body while the PMMA flowed to fill the gaps between fracture fragments. During this step, the less viscous the cement, the more easily it filled defects in the cortex. Additionally, the impaction of the trabecular bone against the surrounding cortical bone reduced the risk of cement penetrating the cortex. Another factor related to the leakage of cement in both VP and $\mathrm{KP}$ is the fracture pattern. When the endplates and anterior or posterior wall are not intact, cement leakage occurs more often [29]. Although the number of cement leakages was small and the results inconclusive, the rate of cement leakage was lowest in the KP with SpineJack group. After expanding the implants, we stabilized the fractured vertebra via injection of PMMA bone cement (Cohesion ${ }^{\circledR}$, Vexim), characterized by its high viscosity; this prevented the occurrence of cement leakage.

It was previously thought that vesselplasty was a safe surgical treatment due to the absence of potentially fatal cement leakage out of the vertebral body and into the spinal canal [30]; however, one of our patients who underwent vesselplasty experienced cement leakage. In a 2015 study, a certain amount of cement leakage occurred during cement injection. This major complication of KP with a vessel resulted in symptomatic, irreversible sequelae, paraplegia due to thermal damage, and spinal nerve compression by the cement, even though an emergent laminectomy was immediately performed [4].

Some data reported rare complications of VP or KP including infections, epidural hemorrhage, fat embolism, cardiac damage, and arterial or renal embolism [31-35]. Fortunately, we did not observe these during the clinical course of our patients.

Although we present promising results, this study had limitations owing to the rapid pace of innovations regarding spinal implants for VCFs. There are some published studies on innovative spinal implants, including the Vertebral Body Stent ${ }^{\circledR}, \mathrm{KIVA}^{\circledR}$ Vertebral Compression Fractures Treatment System, and 
Osseofix ${ }^{\circledR}$. While these implants are useful and are reported to be effective for body height restoration [36], these products have not yet been introduced to our hospital. Therefore, we could not bring these data into our study.

Some researchers might question our choice of implants; however, the patients' economic conditions were considered as well. The treatments used in this study, listed from the most to least expensive, were KP with SpineJack, KP with an IVEP, vesselplasty, balloon KP, and VP. In clinical practice, we emphasized self-decision-making. Thus, we allowed patients to choose between these treatment methods. In addition, SpineJack implants had not been introduced to our hospital until 5 years prior to this report; therefore, all SpineJack data were collected within 5 years. Moreover, we experienced a tragic episode from cement leakage during vesselplasty; since then, we have not had the confidence to perform this procedure again [4]. For this reason, there was no bias of treatment choice based on disease severity; this was a fully randomized retrospective study.

Despite enrolling 354 patients in our study, which is a relatively large patient sample size in a singledoctor series study, our sample size was insufficient to demonstrate the safety of each group. We restricted the study to a single surgeon to minimize the interoperator variability. The safety and effectiveness of surgery depends on the surgeon's technique; nevertheless, though we collected the data from a single surgeon over 10 years, the sample size remained insufficient. Thus, given the small sample size, the result for the least amount of cement leakage remains inconclusive. In further studies, we will enroll more patients to evaluate cement leakage rates in KP more accurately with SpineJack, vesselplasty, and IVEP.

\section{Conclusion}

In summary, our results suggest that VP or KP provide excellent treatment for VCF, with no difference in terms of pain relief. KP with SpineJack had a lower risk of cement extravasation and resulted in greater vertebral body height restoration, kyphotic reduction, and safety than the other procedures, whereas the VP group demonstrated the highest occurrence of adjacent compression fractures. Given the small sample size, the lowest results for cement leakage rate are not conclusive; nevertheless, we recommend that KP with SpineJack, vesselplasty, and IVEP are safe, minimally invasive procedures. Our study will provide surgeons with a reference for choosing a safe and effective procedure for treating VCFs.

\section{Abbreviations}

ANOVA: Analysis of Variance

AVBH average body height

IVEP: intravertebral expandable pillar

KA: kyphotic angle 
KP: kyphoplasty

MRI: magnetic resonance imaging

PMMA: polymethylmethacrylate

SJ: SpineJack

VAS: visual analogue scale

VCF: vertebral compression fractures

VP: vertebroplasty

\section{Declarations}

Ethical approval and consent to participate: Ethical approval was obtained from the institutional review board of Shin-Kong Wu Ho-Su Memorial Hospital, Taiwan (approval number, 20200608R). The requirement for written consent was waived owing to the retrospective study design.

Consent for publication: Not applicable due to the retrospective nature of this study.

Availability of data and material: All data generated or analyzed during this study are included in this published article.

Competing interests: The authors declare that they have no competing interests

Funding: Not applicable.

\section{Authors' contributions:}

Kuei-Lin Yeh contributed the study design, and in the interpretation of data.

Szu-Hsien Wu contributed to the acquisition and analysis of data, and the creation of new software used in the work.

Shing-Sheng Wu assisted in the interpretation of data, and drafted the work

Sheng-Mou Hou contributed to the acquisition and analysis of data, and drafted the work

Acknowledgements: We would like to thank Editage (www.editage.com) for English language editing.

\section{References}


1. Dong R, Chen L, Tang T, Yong Gu, Luo Z, Shi Q, et al. Pain reduction following vertebroplasty and kyphoplasty. Int Orthop. 2013;37:83-7.

2. Cannada LK, Hill BW. Osteoporotic hip and spine fractures: a current review. Geriatr Orthop Surg Rehabil. 2014;5:207-12.

3. Schlaich C, Minne HW, Bruckner T, Wagner G, Gebest HJ, Grunze M, et al. Reduced pulmonary function in patients with spinal osteoporotic fractures. Osteoporos Int. 1998;8:261-7.

4. Yeh KL, Wu S-H, Wu S-S, Chang S-H. Rare episode of cement leakage during vesselplasty in a case of vertebral compression fracture. World Neurosurg. 2020;137:416-20.

5. Xiao H, Yang J, Feng X, Chen P, Li Y, Huang C, et al. Comparing complications of vertebroplasty and kyphoplasty for treating osteoporotic vertebral compression fractures: a meta-analysis of the randomized and non-randomized controlled studies. Eur J Orthop Surg Traumatol. 2015;25:S77-85.

6. Suzuki A, Sekiguchi S, Asano S, Itoh M. Pharmacological topics of bone metabolism: recent advances in pharmacological management of osteoporosis. J Pharmacol Sci. 2008;106:530-5.

7. Eastell R, Rosen CJ, Black DM, Cheung AM, Murad MH, Shoback D. Response to letter to the editor: pharmacological management of osteoporosis in postmenopausal women: an Endocrine Society Clinical Practice Guideline. J Clin Endocrinol Metab. 2019;1:1595-622.

8. Garfin SR, Buckley RA, Ledlie J, Balloon Kyphoplasty Outcomes Group. Balloon kyphoplasty for symptomatic vertebral body compression fractures results in rapid, significant, and sustained improvements in back pain, function, and quality of life for elderly patients. Spine (Phila Pa 1976) 2006;31:2213-20.

9. Liu JT, Liao WJ, Tan WC, Lee JK, Liu CH, Chen YH, et al. Balloon kyphoplasty versus vertebroplasty for treatment of osteoporotic vertebral compression fracture: a prospective, comparative, and randomized clinical study. Osteoporos Int. 2010;21:359-64.

10. Hsieh JY, Wu CD, Wang TM, Chen HY, Farn CJ, Chen PQ. Reduction of the domino effect in osteoporotic vertebral compression fractures through short-segment fixation with intravertebral expandable pillars compared to percutaneous kyphoplasty: a case control study. BMC Musculoskelet Disord. 2013;14:75.

11. Nardi A, Tarantino U, Ventura L, Armotti P, Resmini G, Cozzi L, et al. Domino effect: mechanic factors role. Clin Cases Miner Bone Metab. 2011;8:38-42.

12. Bae JS, Park JH, Kim KJ, Kim HS, Jang IT. Analysis of risk factors for secondary new vertebral compression fracture following percutaneous vertebroplasty in patients with osteoporosis. World Neurosurg. 2017;99:387-94.

13. Firanescu CE, de Vries J, Lodder P, Venmans A, Schoemaker MC, Smeet AJ, et al. Vertebroplasty versus sham procedure for painful acute osteoporotic vertebral compression fractures (VERTOS IV): randomised sham controlled clinical trial. 2018;9:k1551.

14. Theodorou DJ, Theodorou SJ, Duncan TD, Garfin SR, Wong WH. Percutaneous balloon kyphoplasty for the correction of spinal deformity in painful vertebral body compression fractures. Clin Imaging. 2002;26:1-5. 
15. Bouza C, López-Cuadrado T, Cediel P, Saz-Parkinson Z, Amate JM. Balloon kyphoplasty in malignant spinal fractures: a systematic review and meta-analysis. BMC Palliat Care. 2009;8:12.

16. Vanni D, Pantalone A, Bigossi F, Pineto F, Lucantoni D, Salini V. New perspective for third generation percutaneous vertebral augmentation procedures: Preliminary results at 12 months. J Craniovertebr Junction Spine. 2012;3:47-51.

17. Mousavi P, Roth S, Finkelstein J, Cheung G, Whyne C. Volumetric quantification of cement leakage following percutaneous vertebroplasty in metastatic and osteoporotic vertebrae. J Neurosurg. 2003;99:56-9.

18. Berlemann U, Franz T, Orler R, Heini PF. Kyphoplasty for treatment of osteoporotic vertebral fractures: a prospective non-randomized study. Eur Spine J. 2004;13:496-501.

19. Weisskopf M, Herlein S, Birnbaum K, Siebert C, Stanzel S, Wirtz D. [Kyphoplasty - a new minimally invasive treatment for repositioning and stabilising vertebral bodies]. Z Orthop Ihre Grenzgeb. 2003;141:406-11.

20. Adler D, Tschoeke SK, von der Hoeh N, Gulow J, von Salis-Soglio G, Heyde CE. Non-union of osteoporotic vertebral fractures - identification and treatment of an underestimated pathology in elderly patients with persistent back pain. Acta Orthop Belg. 2014;80:444-50.

21. Tsujio T, Nakamura $\mathrm{H}$, Terai $\mathrm{H}$, Masatoshi $\mathrm{H}$, Takashi $\mathrm{N}$, Akira $\mathrm{M}$ et al. Characteristic radiographic or magnetic resonance images of fresh osteoporotic vertebral fractures predicting potential risk for nonunion: a prospective multicenter study. Spine (Phila Pa 1976). 2011;36:1229-35.

22. Krauss M, Hirschfelder H, Tomandl B, Lichti G, Bär I. Kyphosis reduction and the rate of cement leaks after vertebroplasty of intravertebral clefts. Eur Radiol. 2006;16:1015-21.

23. Kim MH, Lee AS, Min SH, Yoon SH. Risk factors of new compression fractures in adjacent vertebrae after percutaneous vertebroplasty. Asian Spine J. 2011;5:180-7.

24. Baroud G, Nemes J, Heini P, Steffen T. Load shift of the intervertebral disc after a vertebroplasty: a finite-element study. Eur Spine J. 2003;12:421-6.

25. Lee IJ, Choi AL, Yie MY, Yoon JY, Jeon EY, Sung Hye et al. CT evaluation of local leakage of bone cement after percutaneous kyphoplasty and vertebroplasty. Acta Radiol. 2010;51:649-654. doi: 10.3109/02841851003620366.

26. Hao J, Hu Z. Percutaneous cement vertebroplasty in the treatment of symptomatic vertebral hemangiomas. Pain Physician. 2012;15:43-49.

27. Nieuwenhuijse MJ, Van Erkel AR, Dijkstra PD. Cement leakage in percutaneous vertebroplasty for osteoporotic vertebral compression fractures: identification of risk factors. Spine J. 2011;11:839-48.

28. Corcos G, Dbjay J, Mastier C, Sandrine L, Auperin A, De Baere T, et al. Cement leakage in percutaneous vertebroplasty for spinal metastases: a retrospective evaluation of incidence and risk factors. Spine (Phila Pa 1976). 2014;39:E332-8.

29. Hsieh MK, Chen LH, Chen WJ. Current concepts of percutaneous balloon kyphoplasty for the treatment of osteoporotic vertebral compression fractures: evidence-based review. Biomed J. 2013;36:154-61.

Page 12/16 
30. Flors L, Lonjedo E, Leiva-Salinas C, Martí-Bonmatí1 L, Martínez-Rodrigo JJ, López-Pérez E, et al. Vesselplasty: a new technical approach to treat symptomatic vertebral compression fractures. AJR Am J Roentgenol. 2009;193:218-26.

31. Iliopoulos $P$, Korovessis $P$, Vitsas V. PMMA embolization to the left dorsal foot artery during percutaneous vertebroplasty for spinal metastases. Eur Spine J. 2014;23:187-91.

32. Prokop A, Hägele M, Pfeilsticker U, Koll S, Chmielnicki M. [Pericardial perforation 2.5 years after kyphoplasty. A rare complication after cement extravasation]. 2013;116:80-4.

33. Ahmadzai H, Campbell S, Archis C, Clark WA. Fat embolism syndrome following percutaneous vertebroplasty: a case report. Spine J. 2014;14:e1-5.

34. Hirata H, Hiwatashi A, Yoshiura T. Resolution of epidural hematoma related to osteoporotic fracture after percutaneous vertebroplasty. World J Radiol. 2013;5:325-7.

35. Chung SE, Lee SH, Kim TH, Yoo KH, Jo BJ. Renal cement embolism during percutaneous vertebroplasty. Eur Spine J. 2006;15:590-4.

36. Cornelis FH, Joly Q, Nouri-Neuville M, Ben-Ammar M, Kastler B, Adrian Kastler A, et al. Innovative spine implants for improved augmentation and stability in neoplastic vertebral compression fracture. Medicina (Kaunas). 2019;55:426

\section{Figures}

\section{Postoperative VAS Score / preoperative VAS score}

1.2

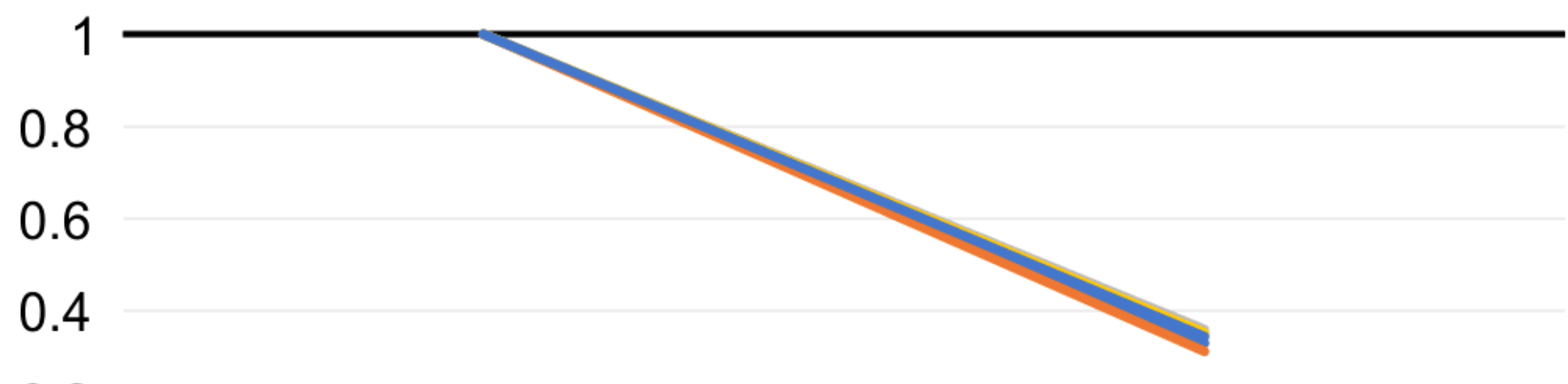

0.2

0

\section{Pre-op} VP - KP-Balloon

\section{2 m post-op}

KP-Vessel - KP-SJ

Figure 1 
Ratio of preoperative VAS score/postoperative VAS score There was no significant different between any of the surgical treatment 1 year postoperatively. VAS, visual analog scale

\section{Postoperative AVBH / preoperative AVBH}

3

2.5

*

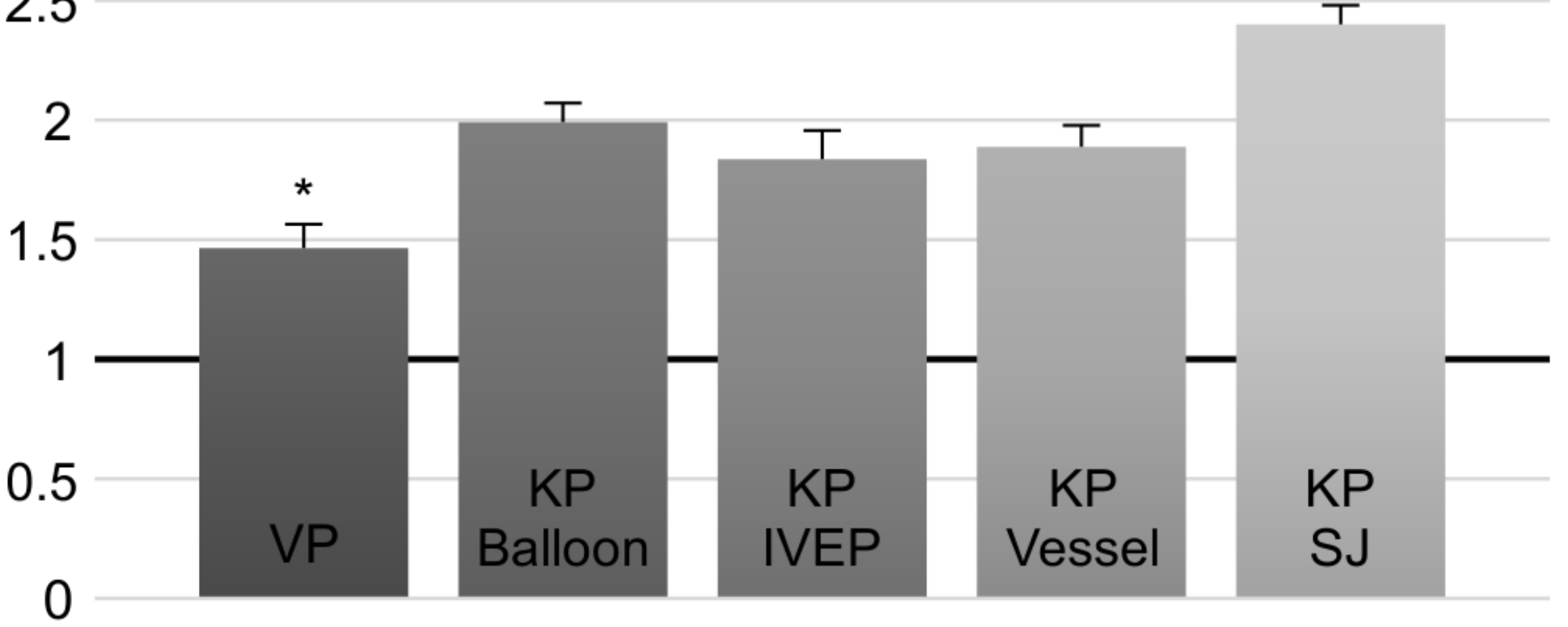

1 yr post-op

Figure 2

Ratio of 1-year postoperative AVBH/preoperative AVBH The vertebroplasty group had the worst performance in terms of AVBH. AVBH, average body height 


\section{Postoperative KA / preoperative KA}

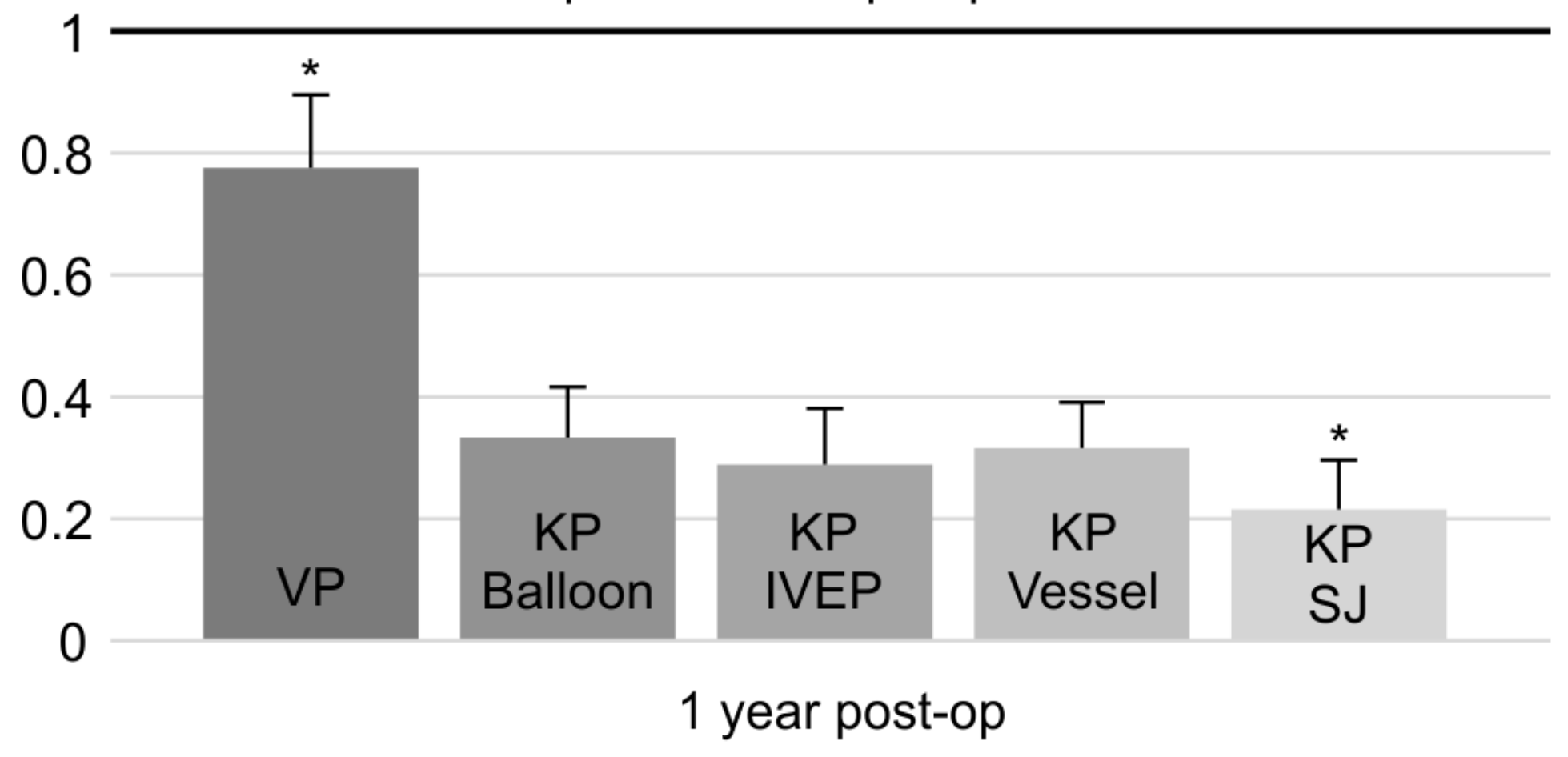

Figure 3

Ratio of 1-year postoperative KA/preoperative KA The vertebroplasty group had the worst performance in terms of KA correction. KA, kyphotic angle

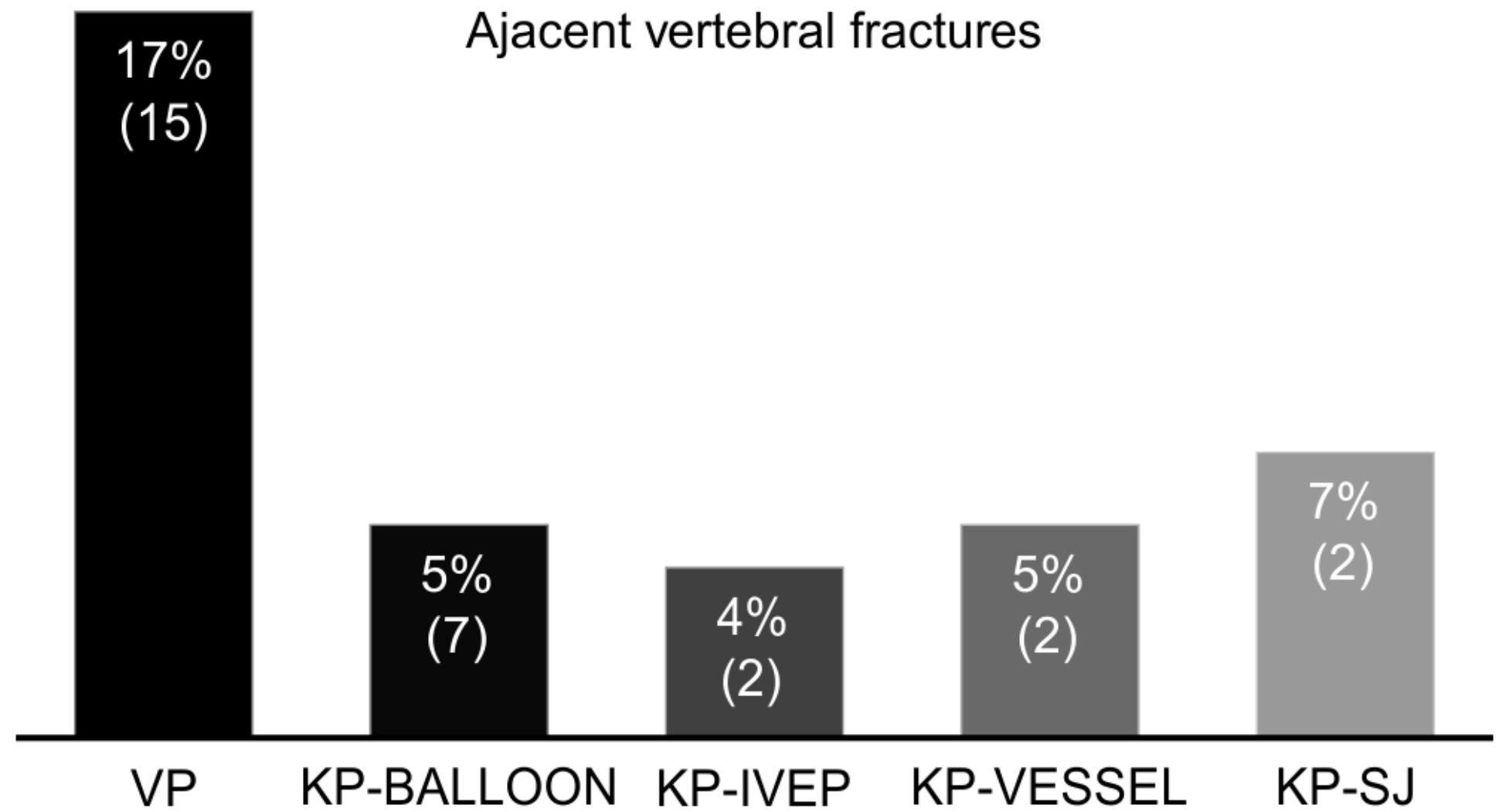

Figure 4 
The rate of adjacent vertebral fracture The highest rate of adjacent vertebral fracture occurred in the vertebroplasty group, with a rate as high as $17 \%$ 